\title{
30 Jahre deutsche Einheit: Wie steht es wirklich?
}

Vor 30 Jahren, am 1. Juli 1990, sind per Staatsvertrag zwei souveräne deutsche Staaten - die Bundesrepublik Deutschland (BRD) und die Deutsche Demokratische Republik (DDR) - eine Währungs-, Wirtschafts- und Sozialunion eingegangen. Zum Jubiläum der Maueröffnung wurde häufig über Unzufriedenheit mancher Bürger der neuen Bundesländer berichtet. Ist dieser Unmut gerechtfertigt? Nach drei Jahrzehnten und geschätzten Kosten von mehr als zwei Billionen Euro liegt die Frage nahe: Kann man bei der Wiedervereinigung von einem ökonomischen Erfolg sprechen? Nach dem Kriterium der ökonomischen Fairness beurteilt sollte man das Einkommen und vor allem den Konsum pro Kopf vergleichen. Demnach genießt der durchschnittliche ostdeutsche Bürger einen Konsum von etwa $90 \%$ des Westniveaus. Um die Kaufkraft berichtigt entspricht das Ost-West-Einkommensgefälle dem zwischen Stadt und Land oder zwischen den Regionen des Nordens und des Südens in Deutschland. Der durchschnittliche westdeutsche Haushalt ist gleichermaßen mit Gebrauchsgütern des täglichen Lebens ausgestattet wie der ostdeutsche. Seit 2004 liegt die Lebenserwartung von Frauen im Osten und Westen auf identischem Niveau; bei Männern hat sich die Lücke bis auf etwa 15 Monate geschlossen. Im Gegensatz zu den anderen ex-sozialistischen Ländern und den USA sind "Tode der Verzweiflung" (durch Suizid und Sucht) rückläufig. Laut jüngsten Befragungen ist die materielle Lebenszufriedenheit im Osten und Westen fast identisch.

Wie passt die hervorragende ökonomische Versorgung der Ostdeutschen zu den nicht selten anzutreffenden Gefühlen, bevormundet zu werden? Ein Hinweis darauf könnte die Entwicklung der realen Ressourcenflüsse geben, also der öffentlichen und privaten Übertragungen, die den Personen im Osten zugute kommen. Ein Maß dieses Ressourcenflusses ist das regionale Bruttoeinkommen abzüglich Absorption (die Summe aus privatem und öffentlichem Konsum sowie Investitionsausgaben). Ein negatives Vorzeichen zeigt, dass per saldo Ressourcen der Region zufließen; ein positiver Wert zeigt das Gegenteil an - typisch für die reichen westdeutschen Bundesländer. Kurz nach der Wende flossen reale Ressourcen umfangreich in den Osten (-40\% des Bruttoeinkommens 1991, -58 \% ohne Berlin), gingen aber ständig zurück - auf -15\% (-19\% ohne Berlin) 2005, und lediglich -4,5\% im Jahr 2016 (etwa -6\% ohne Berlin). Im letzten Jahrzehnt hat der Rückgang wieder an Fahrt aufgenommen und scheint eher privatals staatswirtschaftlich getrieben zu werden.

In der Ökonomie ist man gewohnt, nach Effizienzkriterien zu urteilen. Die Entscheidung, Ressourcen in den Osten zu lenken, hängt mit Zukunftserwartungen von Effizienzgewinnen zusammen. Hier geht es darum, wie effektiv Vorleistungen, Arbeitskräfte und Sachkapital eingesetzt werden, um Güter und Dienstleistungen zu erzeugen - inklusive der Bereitstellung von öffentlichen Gütern wie Umweltqualität und Infrastruktur. Obwohl der Konsum, die Staatsausgaben und die privaten Einkünfte im Osten und Westen vergleichbar sind, werden diese auch durch die Leistung der dortigen Einwohner erwirtschaftet? Gemessen an der Produktivität - Wertschöpfung zu Marktpreisen je Einwohner oder je Stunde - ist die Lage enttäuschend. Natürlich wirkt sich die höhere Erwerbslosenquote im Osten negativ auf die Pro-Kopf-Produktivität aus. Gemessen an der Produktivität je Arbeitsstunde bzw. je Beschäftigten - hinkt

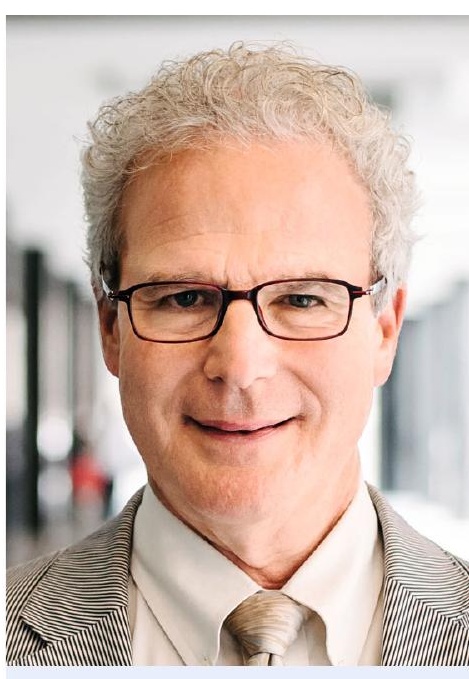

Michael C. Burda ist Lehrstuhlinhaber am Institut für Wirtschaftstheorie II der Humboldt-Universität zu Berlin. 
jedoch der Osten immer noch hinterher, noch verblüffender deswegen, weil die Ostdeutschen mit einer höheren Ausstattung an Sachkapital (bewertet zu Marktpreisen) als ihre Westkollegen arbeiten. Dieser Tatbestand verbirgt politischen Sprengstoff, denn die niedrigere Arbeitsproduktivität ist auch der eigentliche Grund, weshalb die Löhne nach 30 Jahren deutscher Einheit immer noch $15 \%$ unter dem Westniveau liegen. Nicht jede Region eines Landes kann oder soll gleich produktiv sein. In den USA bestehen größere Produktivitätsunterschiede zwischen den Bundesstaaten, die sich jedoch mit der Zeit allmählich abbauen. Man darf aber nicht aus den Augen verlieren, dass Teile Ostdeutschlands vor dem Zweiten Weltkrieg zu den produktivsten Regionen Europas gehörten. 1990 lag die Produktivitätslücke in den neuen Ländern - gemessen am Bruttoinlandsprodukt je Einwohner - bei etwa $70 \%$. Damals hat der US-Ökonom Robert J. Barro gewagt prognostiziert, dass 35 Jahre verstreichen müssten, um die Hälfte der deutsch-deutschen Leistungslücke zu schließen. Im ersten Jahrzehnt nach der Wende wurde seine Prognose widerlegt: der Güterhandel, die Arbeitskräftemobilität, die Sachkapitalinvestitionen und der Transfer von Technologie sorgten dafür, dass bereits 2000 das Produktivitätsniveau erreicht wurde, das erst für 2020 prognostiziert war. Allerdings hat sich seitdem die Ost-West-Konvergenz erheblich verlangsamt. Freilich hat der Osten unter dem Rückgang der Ausrüstungsinvestitionen, dem steigenden Wettbewerbsdruck der neuen EU-Beitrittsländer und Chinas sowie der Finanzkrise gelitten. In fünf Jahren könnte Barros düstere Prognose - wie die Schildkröte, die den Hasen überholt - doch recht bekommen.

Der hartnäckige ostdeutsche Produktivitätsrückstand lässt sich nicht an der Ausbildung oder Fähigkeiten der ostdeutschen Arbeitnehmer festmachen. Früh nach der Wende haben Forscher festgestellt, dass Ostdeutsche, die sich nach der Wende im Westen niederließen, schnell die Produktivität der Kollegen erreichten. An mangelnden Produktionsmitteln liegt es auch nicht: Die ersten zehn Jahre nach der Wende bescherten den Ostdeutschen eine bessere Kapitalausstattung als den Westkollegen. Es bleibt nur noch die Gesamtfaktorproduktivität (total factor productivity, TFP), also jene Effektivität, die beim Einsatz von vergleichbaren Arbeitskräften und Produktionsmitteln erreichbar ist. Zunächst konvergierte die TFP durchaus stark, aber seit 2000 ist sie bei der Hälfte des Westens stehengeblieben. Nur in der Landwirtschaft liegt die TFP im Osten höher. Die TFP wird von mehreren Faktoren mitbestimmt. Zu den „harten“ Determinanten gehören moderne Technologien, die Industriestruktur, die Qualität der Führungskräfte, die Preise der erzeugten Wertschöpfung sowie die Aktivitäten in Forschung und Entwicklung. „Weiche“ Faktoren sind unternehmerisches Vertrauen und Unternehmerkultur, Gründergeist und Firmennetzwerke. Ursachenforschung dazu deutet auf innerbetriebliche Vorleistungen, Managerdichte, Neugründungen und Standorte von Großkonzernen. Aber neue Erkenntnisgewinne werden durch die Verfügbarkeit von belastbaren Daten beschränkt.

Immer noch wird spekuliert, ob man die volkswirtschaftliche Transformation des Ostens anders und besser hätte gestalten können. Viele Vorschläge - eine „Sonderwirtschaftszone Ost“" mit niedrigen bzw. subventionierten Löhnen, gemindertem Mehrwertsteuersatz oder sogar mit abgewertetem Wechselkurs, wären nicht politisch durchsetzbar gewesen und hätten wohl zu einem deutschen Mezzogiorno geführt. Der Fairness wegen hätte mehr Wohneigentum an Ostdeutsche gegeben werden können. Damit hätte man die ungleiche Vermögensverteilung, aber keineswegs die Ursachen der Ungleichheit, gelindert. Trotz des Produktivitätsgefälles zwischen Ost und West genießen die Ostdeutschen unbestritten verbesserte Lebensstandards und eine freiheitliche Wirtschafts- und Sozialordnung. Nach 30 Jahren legen die systematischen

Michael C. Burda

Humboldt-Universität zu Berlin burdamic@cms.hu-berlin.de
Ost-West-Produktivitätsunterschiede entlang der alten BRD-DDR-Grenze nahe, dass die Trennung Deutschlands zu einer hartnäckigen Vernarbung geführt hat, die es wissenschaftlich weiterhin zu untersuchen und zu verstehen gilt. 